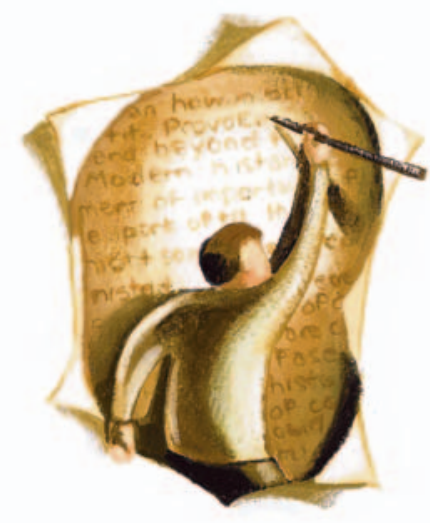

\section{CMA driver's guide}

I have concerns about the CMA's guidelines on determining medical fitness to drive. ${ }^{1}$ All Canadian provinces and territories currently use the medical standards produced by the Canadian Council of Motor Transport Administrators (CCMTA) ${ }^{2}$ to determine the medical fitness of drivers. There are considerable discrepancies between the CMA and CCMTA guidelines, resulting in confusion for both clinicians and patients. For example, according to the CMA driver's guide, someone who has experienced a single unprovoked seizure requires a neurological assessment, an electroencephalogram (awake and asleep), neuroimaging (CT scan or MRI) and a seizure-free period of at least 3 months before returning to driving. In contrast, the CCMTA guidelines state that a neurological assessment and an electroencephalogram would be required before this person could drive again, with no seizure-free period specified if the electroencephalogram is normal. Although the CMA driver's guide does state that provincial and territorial motor vehicle licensing authorities follow CCMTA standards, the CMA does not present the CCMTA standards in its driver's guide, nor does it document where its guidelines differ from the CCMTA standards or provide justification for these differences.

The CMA driver's guide correctly states that "only motor vehicle licensing authorities can suspend or restrict a person's licence" and "it is not the physician's responsibility to determine whether the patient's driving privileges should be altered." It is also important to recognize that it is not the CMA's responsibility to determine whether a person's driving privileges should be altered. I agree that the CMA should have a role in setting guidelines for determining the medical fitness of drivers, but the association should interact directly with the CCMTA and not disseminate a set of guidelines that do not accurately reflect the standards that are used by licensing authorities to determine medical fitness to drive.

\section{Donald W. Gross MD \\ Director \\ Comprehensive Adult Epilepsy \\ Program \\ University of Alberta \\ Edmonton, Alta.}

\section{REFERENCES}

I. Canadian Medical Association. Determining medical fitness to operate motor vehicles. CMA driver's guide. 7 th ed. Ottawa $(\mathrm{ON})$ : The Association; 2006.

2. Canadian Council of Motor Transport Administrators. CCMTA medical standards for drivers. Ottawa (ON): The Council; 2006.

DOI:I0.1503/cmaj.1070037

\section{[The editor-in-chief of the CMA}

\section{driver's guide responds:]}

Donald Gross raises an important issue that was discussed in depth by the Scientific Editorial Board for the 7 th edition of the CMA driver's guide. ${ }^{1}$ In many instances, the regulations produced by the CCMTA, which are used as the basis for provincial and territorial licensing standards, do not apply to a particular patient's situation, or relevant regulations do not exist. The goal of the CMA driver's guide is to assist physicians in determining the medical fitness of such patients to drive; the guide has a particular emphasis on the functional assessment of patients with multiple medical conditions.

The CCMTA standards are based on eligibility criteria to hold licensure to operate different classes of motor vehicles, with no reference to the context of driving. The CMA driver's guide helps physicians to consider the context of driving for individual patients. This distinction has created some conflicts between the CCMTA standards and the CMA driver's guide. For example, the CMA driver's guide establishes different guidelines for private or commercial drivers based on the amount and circumstances of driving; the CCMTA standards only make a distinction between private and commercial driving in terms of the type of vehicle driven. The CMA driver's guide enables physicians to advise their patients on safe driving or driving cessation independent of their legal permit to drive, as well as providing an evidence base, where one exists, that physicians can use to advocate for relicensure for their patients on the basis of their individual functional capacity.

The neurological standards cited by Gross are an example of the distinction between the admissibility criteria for licensing and the guidelines provided for physicians to use in assessing their patients. The CMA guidelines are based on research evidence, the opinion of physicians recognized as experts in a particular field or the consensus opinion of the relevant specialty society. In many instances the CCMTA has used CMA guidelines and consensus statements from specialty groups, on the advice of its Medical Advisory Committee, to establish licensing standards. However, not all medical practice guidelines can be easily translated into regulatory standards.

The CCMTA and the CMA communicate closely in the production of both the CCMTA licensing standards and the CMA driver's guide. The current and past chairs of the CCMTA Medical Advisory Committee served on the Scientific Editorial Board for the 7 th edition of the CMA driver's guide, and the CMA has long had a representative at the CCMTA Medical Advisory Committee's meetings. In many instances, this 